\title{
Beiträge zur Kenntnis der biologischen Wirkung des Broms.
}

\author{
III.
}

Wirkung des Broms auf die Zirkulation bei Kaninchen.

\author{
Von \\ Saburo Tada. \\ (多田三郎) \\ (Aus der Medizinischen Krlinili von Prof. Dr. T. Kato, \\ Káciserliche Lriversität an Senddu.)
}

Einleitung und Versuchsmethodik.

Die Wirkung von Bromverbindungen auf das Zirkulationssystem ist noch strittig.

Eulenburg und, Guttmann behaupten, dass Bromkalium die Herzaktion rerlangsamt, was nicht ron der Vagusreizung, sondern ron der Lïhmung der Herzmuskeln herrühren soll. K ros z ${ }^{2}$ iussert sich dahin, dass die durch Injektion ron Bromkalium hervorgerufene Pulsverlangcamung nicht auf Brom, sondern auf dem Kaliumkomponenten beruht, und $\mathrm{G}$ reen e und $\mathrm{K}$ ruse $\mathrm{e}^{3)}$ meinen, dass bei der Durchspülung eines isolierten Warmblüterherzens mit Ringerlösung, wenn sie auf eine an Stelle des $\mathrm{NaCl}$ durch $\mathrm{NaBr}$ ersetate Lösung umgeschaltet wird, die Herzschlïge in den meisten Füllen verlangsamt und die Amplitude rerkleinert wird, welche Wirkung sie darauf zuriickfiihren, dass $\mathrm{Br}$ die Koronargefïsse verengert und dadurch die Herzmuskeln angreift. Yoshitom it) teilt mit, dass bei der Durchströmung eines isolierten Froschberzens nach der Stra ubschen Methode mit Ringer, wenn sie gegen eine statt des Cl-Komponenten substituiertes Brom enthaltende Ringerlösung umgetauscht wird, in diesem Augenblicke die Systole auffallend und die Diastole nur unbedeutend abnimmt, diese jedoch bald zur Norm zurückkehrt, während jene allmühlich stïrker wird, bis sich schliesslich die Amplitude und Frequenz vermehren im Vergleich mit denen bej der Durchspülung mit Ringer, und dass diese Verïnderungen daher rïhren, dass durch $\mathrm{Br}$ die Erregbarkeit der Herzmuskeln gesteigert wird. Finck $\mathrm{h}^{\text {j) }}$ fand bei der Durchströmung eines Froschherzens nach dem Stra ubschen Verfahren, dass auch bei dem Umschalten der Durchspülungsflüssigkeit auf eine solche, in der das $\mathrm{NaCl}$ der Ringer durch $\mathrm{NaBr}$ oder das sümtliche $\mathrm{Cl}$ durch Br ersetzt ist, die IIerztiitigkeit gar nicht geschädigt wird.

1) Eulenburg u. Guttmann, Virchow's Arch., 1\$67, 41, 91.

2) K ros z, Arch. f. exp. Pathol. u. Pharm., 1877, 6, 1.

3) Greene, u. Kruse, Journ. Americ. Med. Assoc., 1913, 271.

4) Yoshitom i, Kyoto-Igaku-Zasshi, 1920, 17, 618. (jap.)

5) Fin ck h, Biochem. Zeitschr., 1921, 116, 262. 
So scheint die Bromverbindung das Warmblüterherz herabzusetzend, aber am Froschherzen hat man noch keinc eindeutigen Resultate erhalten.

Die Untersuchungen über die Gefïsswirkung der Bronverbindung scheinen nur selten angestellt worden zu sein. Neuerdings hat Yoshito $\mathrm{mi}^{4}$ ) bei der Durchströmung von Froschschenkel und Kaninchenohr mit Ringerlösung, in der $\mathrm{Cl}$ durch Br ersetzt war, geringe Gefüsserweiterung konstatiert, woraus er den Schluss zog, dass dieses auf die Gefässmuskeln lïhmend wirkt. Auch Guggenheimer und Fisher $\left.{ }^{6}\right)$ haben gefunden, dass durch Versetzen der Durchströmungstlüssigkeit mit einer kleinen Dose $\mathrm{NaBr}$ die Koronararterien des isolierten Herzens und die peripheren Gefïsse von Katzen dilatiert werden.

Ich $^{72}$ wies bei Kaninchen die Hemmung des Sauerstoffverbrauchs des Gesamtorganismus sowie der Sauerstoffzehrung der Erythrozyten durch Brom nach. Da sich bei der Veränderung des Gaswechsels des Organismus die Blutströmung auch ändern würde, so habe ich in der vorliegenden Arbeit die Veründerung des Minntenvolums durch Bromgabe vermittelt, um den Einfluss dieses Halogens auf die Dynamik des Zirkulationssystems zu beobachten.

Zum Versuche dienten, wie bei der Untersuchung der Bromwirkung auf den Sauerstoff verbrauch, ${ }^{7}$ reife Kaninchen. Die Messung des Minutenvolums erfolgte wie bei Odaira ${ }^{8)}$ nach dem Prinzip von Fick.9)

Das venöse Blut wurde durch Punktion vorsichtig aus dem rechten Herzen und das arterielle aus der A. femoralis entnommen. An dem so gewonnenen Blut wurde sofort mittels des Barcroftschen Differentialblutgasapparats ${ }^{10)}$ der Sauerstoffgehalt bestimmt. Der Sauerstoffverbrauch des Kaninchens wurde mittels des Roth schen Apparats ermittelt. Die zu injizierende Chlor- bzw. Bromnatriumlösung war stets hy pertonisch und miteinander molekül-äquivalent, d. h. 10\% für $\mathrm{NaCl}$ und 17,61\% für $\mathrm{NaBr}$, betrug pro kg Körpergewicht $5 \mathrm{ccm}$. Sie wurde körperwarm und langsam gleichmässig in die V. jugularis eingespritzt.

\section{Versuchsergebnisse.}

Zuerst wurde zur Kontrolle eine 10\%ige NaCl-Lösung intravenös injiziert und die dadurch hervorgerufenen Veränderungen des Minutenvolums beobachtet (Tab. 1). u. 114 .

6) Guggenheimer u. Fisher, Arch. f. exp. Pathol. u. Pharm., 1927, 126, 104

7) Tada, Tohoku Journ. Exp. Med., 1930, 15, 236.

8) Odaira, Tohoku Journ. Exp. Med., 1925, 6, 325. 118,361 .

9) Fick, Würzburg 1870. Zit. n. Lundsgaard, Dtsch. Arch. f. klin. Med., 1916,

10) Barcroft, The respiratory function of the blood, Cambridge 1914. 
'Tabelle 1.

Veränderung des Minutenvolums durch Chlornatrium.

\begin{tabular}{|c|c|c|c|c|c|c|c|c|c|}
\hline \multirow[b]{2}{*}{ Nr. } & \multirow{2}{*}{$\begin{array}{l}\text { Gewicht } \\
\text { (kg) u. } \\
\text { Geschl. }\end{array}$} & \multirow{2}{*}{$\begin{array}{l}\text { Dose } \\
\text { (g) d. } \\
\mathrm{NaCl}\end{array}$} & \multirow[b]{2}{*}{ Zeit } & \multirow{2}{*}{$\begin{array}{l}\text { Sauerstoff- } \\
\text { verbrauch } \\
\text { pro Min. } \\
\text { (cem) }\end{array}$} & \multirow[b]{2}{*}{$\mathrm{O}_{1}-\mathrm{O}_{\mathrm{V}}$} & \multicolumn{2}{|c|}{ Minutenrolum } & \multirow{2}{*}{ Puls } & \multirow{2}{*}{ Resp. } \\
\hline & & & & & & $(\mathrm{cm})$ & $\begin{array}{l}\text { Verïn- } \\
\text { derung } \\
\text { in } 0 ; 0\end{array}$ & & \\
\hline 1 & 1,7 & 0,85 & 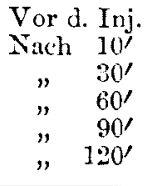 & $\begin{array}{l}18,4 \\
18,6 \\
18,8 \\
18,3 \\
18,8 \\
19,2\end{array}$ & $\begin{array}{l}0,0732 \\
0,0674 \\
0,0712 \\
0,0730 \\
0,0743 \\
0,0756\end{array}$ & $\begin{array}{l}251 \\
276 \\
264 \\
251 \\
253 \\
254\end{array}$ & $\begin{array}{r}+10,0 \\
+\quad 5,2 \\
+\quad 0 \\
+\quad 0,8 \\
+1,2\end{array}$ & $\begin{array}{l}270 \\
264 \\
276 \\
270 \\
276 \\
270\end{array}$ & $\begin{array}{l}102 \\
102 \\
108 \\
102 \\
108 \\
108\end{array}$ \\
\hline 2 & 1,8 & 0,9 & $\begin{array}{l}\text { Vor d. Inj. } \\
\text { Nach } \quad 10^{\prime} \\
\quad " \quad 30^{\prime} \\
\Rightarrow \quad 60^{\prime} \\
\Rightarrow \quad 90^{\prime} \\
\Rightarrow \quad 120^{\prime}\end{array}$ & $\begin{array}{l}23,3 \\
23,5 \\
23,9 \\
23,0 \\
24,1 \\
24,9\end{array}$ & $\begin{array}{l}0,0933 \\
0,0879 \\
0,0920 \\
0,0931 \\
0,0935 \\
0,0949\end{array}$ & $\begin{array}{l}250 \\
267 \\
260 \\
247 \\
258 \\
262\end{array}$ & $\begin{array}{l}+6,8 \\
+\quad 4,0 \\
-1,2 \\
+\quad 3,2 \\
+\quad 4,8\end{array}$ & $\begin{array}{l}240 \\
216 \\
222 \\
222 \\
240 \\
234\end{array}$ & $\begin{array}{l}\$ 4 \\
90 \\
90 \\
84 \\
90 \\
90\end{array}$ \\
\hline 3 & 2,0 & 1,0 & $\begin{array}{l}\text { Vor d. Inj. } \\
\text { Nach } \quad 10^{\prime} \\
\quad " \quad 30^{\prime} \\
\Rightarrow \quad 60^{\prime} \\
\Rightarrow \quad 90^{\prime} \\
\Rightarrow \quad 120^{\prime}\end{array}$ & $\begin{array}{l}25,8 \\
25,8 \\
26,2 \\
26,0 \\
27,5 \\
27,8\end{array}$ & $\begin{array}{l}0,1034 \\
0,0935 \\
0,1030 \\
0,1036 \\
0,1050 \\
0,1076\end{array}$ & $\begin{array}{l}250 \\
276 \\
254 \\
251 \\
262 \\
258\end{array}$ & $\begin{array}{l}+10,4 \\
+\quad 1,6 \\
+0,4 \\
+\quad 4,8 \\
+3,2\end{array}$ & $\begin{array}{l}264 \\
258 \\
258 \\
264 \\
264 \\
252\end{array}$ & $\begin{array}{l}82 \\
90 \\
90 \\
96 \\
96 \\
90\end{array}$ \\
\hline 4 & $1,85 \quad 3$ & 0,925 & $\begin{array}{c}\text { Vor d. Inj. } \\
\text { Nach } 10^{\prime} \\
\text { " } \quad 30^{\prime} \\
\Rightarrow \quad 60^{\prime} \\
" \quad 90^{\prime} \\
\Rightarrow \quad 120^{\prime}\end{array}$ & $\begin{array}{l}24,0 \\
24,2 \\
24,6 \\
24,0 \\
26,6 \\
26,0\end{array}$ & $\begin{array}{l}0,0 S 92 \\
0,0817 \\
0,0880 \\
0,0882 \\
0,0940 \\
0,0942\end{array}$ & $\begin{array}{l}269 \\
296 \\
280 \\
272 \\
283 \\
276\end{array}$ & $\begin{array}{l}+10,0 \\
+4,1 \\
+1,1 \\
+5,2 \\
+\quad 2,6\end{array}$ & $\begin{array}{l}258 \\
258 \\
258 \\
258 \\
270 \\
270\end{array}$ & $\begin{array}{l}60 \\
54 \\
60 \\
54 \\
60 \\
66\end{array}$ \\
\hline 5 & $2,3 \quad$ P & 1,15 & $\begin{array}{l}\text { Vor d. Inj. } \\
\text { Nach } \quad 10^{\prime} \\
\quad, \quad 30^{\prime} \\
" \quad 60^{\prime} \\
" \quad 90^{\prime} \\
\Rightarrow \quad 120^{\prime}\end{array}$ & $\begin{array}{l}21,5 \\
22,0 \\
21,6 \\
21,5 \\
22,2 \\
23,1\end{array}$ & $\begin{array}{l}0,0711 \\
0,0657 \\
0,0687 \\
0,0708 \\
0,0705 \\
0,0726\end{array}$ & $\begin{array}{l}302 \\
335 \\
314 \\
304 \\
315 \\
315\end{array}$ & $\begin{array}{l}+10,9 \\
+\quad 4,0 \\
+\quad 0,7 \\
+\quad 4,3 \\
+\quad 5,3\end{array}$ & $\begin{array}{l}270 \\
270 \\
264 \\
270 \\
270 \\
270\end{array}$ & $\begin{array}{l}72 \\
72 \\
72 \\
72 \\
72 \\
72\end{array}$ \\
\hline
\end{tabular}

Aus Tab. 1 ersieht man, dass das Minutenvolum zehn Minuten nach der Injektion der NaCl-Lösung immer zugenommen hat, und zwar um 6,8-10,9\% von vor der Injektion. Diese Zunahme wird allmählich kleiner, um nach einer Stunde im allgemeinen Neigung zur W iederherstellung zu zeigen, und $1 \frac{1}{2}-2$ Stunden nach der Injektion nimmt es wieder etwas zu.

Nun wurden die Veränderungen des Minutenvolums nach intravenöser Injektion einer der 10\% igen $\mathrm{NaCl}$-Lösung isotonischen, d. h. 17,61 \%igen, $\mathrm{NaBr}$-Lösung ermittelt (Tab. 2). 
Fig. 1.

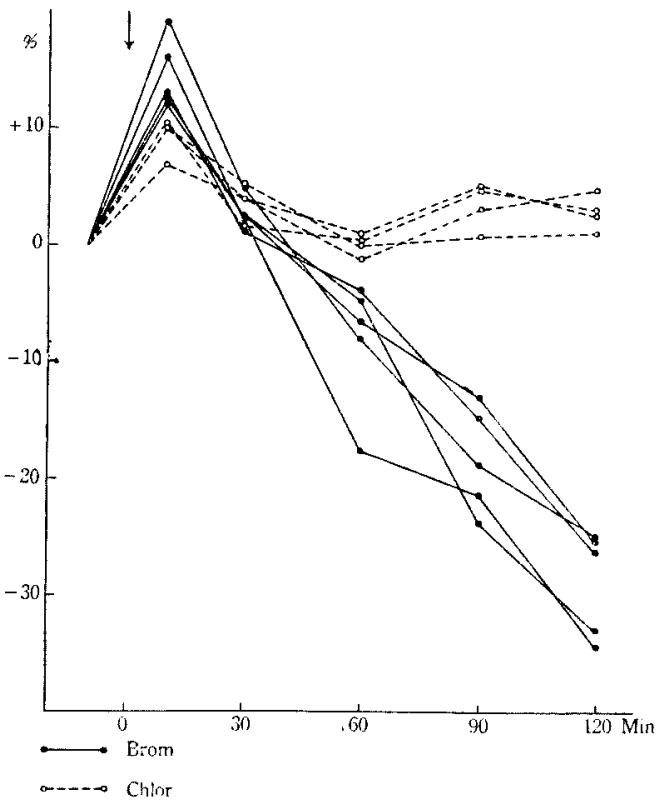

Zu- und Abnahme des Minutenvolums (in \%) durch Injektion einer hypertonischen Brom- bzw. Chlornatriumlösung. Bei $\downarrow$ injiziert.
Aus Tab. 2 sieht man, dass in allen Fällen das Minutenvolum immer zehn Minuten post injectionem der Bromnatriumlösung urn 11,9$19,0 \%$ gegenüber dem vor der Injektion zugenommen hat, was zwar die gleiche Veränderung wie beim $\mathrm{NaCl}$ darstellt, aber immer stärker ist; nach einer halben Stunde zeigt es eine, wenn auch geringe, Zunahme, aber nach 1 Stunde nimmt es ab und in $1 \frac{1}{2}-2$ Stunden immer bedeutender; dies trifft ja beim $\mathrm{NaCl}$ nicht zu.

\section{Tabelle 2.}

Verïnderung des Minutenvolums dureh Bromnatrium.

\begin{tabular}{|c|c|c|c|c|c|c|c|c|c|}
\hline \multirow[b]{2}{*}{ Nr. } & \multirow{2}{*}{$\begin{array}{l}\text { Gewicht } \\
\text { (kg) u. } \\
\text { Geschl. }\end{array}$} & \multirow{2}{*}{$\begin{array}{l}\text { Dose } \\
\text { (g) d. } \\
\text { NaBr }\end{array}$} & \multirow[b]{2}{*}{ Zeit } & \multirow{2}{*}{$\begin{array}{l}\text { Sauerstoff- } \\
\text { verbrauch } \\
\text { pro Min. } \\
\text { (ccm) }\end{array}$} & \multirow[b]{2}{*}{$\mathrm{O}_{\lambda}-\mathrm{O}_{V}$} & \multicolumn{2}{|c|}{ Minutenvolum } & \multirow{2}{*}{ Puls } & \multirow{2}{*}{ Resp. } \\
\hline & & & & & & (cem) & $\begin{array}{l}\text { Verün- } \\
\text { derung } \\
\text { in } \%\end{array}$ & & \\
\hline 1 & 1,9 & 1,67 & $\begin{array}{c}\text { Vor d. Inj. } \\
\text { Nach } \\
\quad 30^{\prime} \\
\Rightarrow \quad 30^{\prime} \\
\Rightarrow \quad 60^{\prime} \\
\Rightarrow \quad \\
\Rightarrow \quad 120^{\prime}\end{array}$ & $\begin{array}{l}22,8 \\
22,8 \\
23,0 \\
20,8 \\
17,8 \\
16,8\end{array}$ & $\begin{array}{l}0,0965 \\
0,0865 \\
0,0950 \\
0,0923 \\
0,0989 \\
0,1063\end{array}$ & $\begin{array}{l}236 \\
264 \\
242 \\
225 \\
180 \\
158\end{array}$ & $\begin{array}{l}+11,9 \\
+2,5 \\
-4,7 \\
-23,7 \\
-33,1\end{array}$ & $\begin{array}{l}252 \\
234 \\
240 \\
240 \\
234 \\
228\end{array}$ & $\begin{array}{l}60 \\
60 \\
60 \\
54 \\
54 \\
48\end{array}$ \\
\hline 2 & 2,0 & 1,76 & $\begin{array}{c}\text { Vor d. Inj. } \\
\text { Nach } \quad 10^{\prime} \\
\quad " \quad 30^{\prime} \\
\Rightarrow \quad 60^{\prime} \\
\Rightarrow \quad 90^{\prime} \\
\Rightarrow \quad 120^{\prime}\end{array}$ & $\begin{array}{l}25,7 \\
26,1 \\
26,2 \\
23,8 \\
21,8 \\
20,4\end{array}$ & $\begin{array}{l}0,1020 \\
0,0870 \\
0,0992 \\
0,1026 \\
0,1063 \\
0,1080\end{array}$ & $\begin{array}{l}252 \\
300 \\
264 \\
232 \\
205 \\
189\end{array}$ & $\begin{array}{r}+19,0 \\
+\quad 4,8 \\
-7,9 \\
-18,7 \\
-25,0\end{array}$ & $\begin{array}{l}276 \\
270 \\
276 \\
270 \\
258 \\
258\end{array}$ & $\begin{array}{l}54 \\
54 \\
48 \\
48 \\
48 \\
48\end{array}$ \\
\hline
\end{tabular}




\begin{tabular}{|c|c|c|c|c|c|c|c|c|c|}
\hline \multirow[b]{2}{*}{ Nr. } & \multirow[b]{2}{*}{$\begin{array}{c}\text { Gewicht } \\
\text { (kg) u. } \\
\text { Geschl. }\end{array}$} & \multirow[b]{2}{*}{$\begin{array}{l}\text { Dose } \\
(\mathrm{g}) \mathrm{d} . \\
\mathrm{NaBr}\end{array}$} & \multirow[b]{2}{*}{ Zuit } & \multirow{2}{*}{$\begin{array}{l}\text { Sauerstoff- } \\
\text { verbrauch } \\
\text { pro Min. } \\
\text { (ccm) }\end{array}$} & \multirow[b]{2}{*}{$\mathrm{O}_{\lambda}-\mathrm{O}_{r}$} & \multicolumn{2}{|c|}{ Minutenvolum } & \multirow{2}{*}{ Puls } & \multirow{2}{*}{ Resp. } \\
\hline & & & & & & $(\mathrm{cm})$ & $\begin{array}{l}\text { Verün- } \\
\text { derung } \\
\text { in } 0 ;\end{array}$ & & \\
\hline 3 & 1,6 & 1,4 & $\begin{array}{l}\text { Vor d. Inj. } \\
\text { Nach } \quad 10^{\prime} \\
\Rightarrow \quad 30^{\prime} \\
" \quad \quad 60^{\prime} \\
\Rightarrow \quad 90^{\prime} \\
\Rightarrow \quad 120^{\prime}\end{array}$ & $\begin{array}{l}16,4 \\
16,6 \\
16,6 \\
14,1 \\
13,7 \\
11,7\end{array}$ & $\begin{array}{l}0,0712 \\
0,0622 \\
0,0707 \\
0,0743 \\
0,0757 \\
0,0777\end{array}$ & $\begin{array}{l}230 \\
267 \\
235 \\
190 \\
181 \\
151\end{array}$ & $\begin{array}{l}+16,1 \\
+\quad 2,2 \\
-17,4 \\
-21,3 \\
-34,3\end{array}$ & $\begin{array}{l}228 \\
216 \\
234 \\
216 \\
216 \\
210\end{array}$ & $\begin{array}{l}66 \\
60 \\
60 \\
60 \\
66 \\
66\end{array}$ \\
\hline 4 & 1,6 & 1,4 & $\begin{array}{c}\text { Vor d. Inj. } \\
\text { Nach } \quad 10^{\prime} \\
\Rightarrow \quad 30^{\prime} \\
" \quad 60^{\prime} \\
\Rightarrow \quad 90^{\prime} \\
\Rightarrow \quad 120^{\prime}\end{array}$ & $\begin{array}{l}13,5 \\
13,9 \\
14,0 \\
12,8 \\
12,4 \\
10,9\end{array}$ & $\begin{array}{l}0,0688 \\
0,0615 \\
0,0680 \\
0,0680 \\
0,0708 \\
0,0727\end{array}$ & $\begin{array}{l}201 \\
226 \\
206 \\
188 \\
175 \\
150\end{array}$ & $\begin{array}{l}+12,4 \\
+\quad 2,5 \\
-6,5 \\
-12,9 \\
-25,4\end{array}$ & $\begin{array}{l}204 \\
192 \\
204 \\
204 \\
198 \\
192\end{array}$ & $\begin{array}{l}66 \\
54 \\
60 \\
60 \\
60 \\
60\end{array}$ \\
\hline 5 & $1,65 ?$ & 1,45 & $\begin{array}{l}\text { Vor d. Inj. } \\
\text { Nach } 10^{\prime} \\
\quad " \quad 30^{\prime} \\
\Rightarrow \quad 60^{\prime} \\
\Rightarrow \quad 90^{\prime} \\
\Rightarrow \quad 120^{\prime}\end{array}$ & $\begin{array}{l}26,6 \\
26,7 \\
27,1 \\
25,9 \\
24,5 \\
23,7\end{array}$ & $\begin{array}{l}0,0907 \\
0,0807 \\
0,0912 \\
0,0918 \\
0,0980 \\
0,1096\end{array}$ & $\begin{array}{l}293 \\
331 \\
297 \\
282 \\
250 \\
216\end{array}$ & $\begin{array}{l}+18,0 \\
+\quad 1,4 \\
-3,8 \\
-14,7 \\
-26,3\end{array}$ & $\begin{array}{l}270 \\
264 \\
282 \\
270 \\
264 \\
258\end{array}$ & $\begin{array}{l}96 \\
96 \\
96 \\
94 \\
90 \\
90\end{array}$ \\
\hline 6 & 1,7 & 1,5 & $\begin{array}{c}\text { Vor d. Inj. } \\
\text { Nach } 10^{\prime} \\
\text { " } \quad 30^{\prime} \\
\Rightarrow \quad 60^{\prime} \\
\Rightarrow \quad 90^{\prime} \\
\Rightarrow \quad 120^{\prime}\end{array}$ & $\begin{array}{l}19,2 \\
19,5 \\
19,5 \\
18,8 \\
16,6 \\
15,8\end{array}$ & $\begin{array}{l}0,0733 \\
0,0628 \\
0,0722 \\
0,0723 \\
0,0786 \\
0,0814\end{array}$ & $\begin{array}{l}262 \\
311 \\
270 \\
260 \\
211 \\
194\end{array}$ & $\begin{array}{l}+18,7 \\
+3,1 \\
-0,8 \\
-19,5 \\
-26,0\end{array}$ & $\begin{array}{l}282 \\
258 \\
282 \\
270 \\
270 \\
264\end{array}$ & $\begin{array}{l}66 \\
66 \\
66 \\
66 \\
66 \\
66\end{array}$ \\
\hline
\end{tabular}

\section{Zusammenfassung und Besprechung.}

Von Dastre und Loye, ${ }^{11}$ Leathes, ${ }^{12}$ Smith und Mende ${ }^{13}$ and $\mathrm{Nait}{ }^{14)}$ wurde festgestellt, dass bei intravenöser Injektion einer hypertonischen Lösung die im Kreislauf zirkulierende Flüssigkeit vorübergehend zunimmt. Die Vermehrung des Minutenvolums unmittelbar nach intravenöser Finspritzung von Chlor- oder Bromanatriumlösung in meinen Versuchen rührt von der Zunahme des Gesamtblutvolums her. Bei seiner Znnahme wird die Herzaktion gesteigert, was zur Zunahme des Sauerstoffverbrauchs und des Minutenvolums führt. In meinen Versuchen beruht die Zunahme des Minutenvolums auf den oben geschilderten zwei Faktoren. Doch nimmt, wie ersichtlich, bei der Injektion von Bromnatriumlösung das Minu-

11) Dastre u. Loye, Arch. de Physiol. et Path., 1888-9, 2, 73 u. 253.

12) Leathes, Journ. Physiol., 1895-96, 19, I.

13) Smith u. Mendel, Americ. Journ. Physiol., 1920, 53, 323.

14) Naito, Tohoku Journ. Exp. Med., 1925-25, 5, 351. 
tenvolum in allen Fïllen stärker zu als bei der von Chlornatriumlösung. Es fragt sich nun, woher dieser Unterschied kommt. Betr. der Gefüsswirkung der Bromverbindung fand, wie schon erwähnt, Yoshitomi ${ }^{4}$ an Froschschenkel- und Kaninchenohrgefïssen und Guggenheimer und Fisher $\mathbf{r}^{6)}$ an Koronargefüssen und peripheren Gefässen von Katzen, dass Brom vasodilatatorisch wirkt. Um die Resultate obiger Autoren zu prüfen und damit einen Beitrag zum Minutenvolum zu liefern, habe ich folgenden Versuch angestellt:

Zuerst wurden das isolierte Schenkel- und Ohrgefüss von Kaninchen mit normaler Ringer durchströmt und diese beim Konstantwerden des Ausflusses auf eine solche, bei der $\mathrm{Cl}$ durch $\mathrm{Br}$ substituiert war, umgeschaltet, und nun die dadurch auftretenden Veränderungen beobachtet. Die hier gebrauchte normale Ringer bestand aus $0,9 \% \mathrm{NaCl}, 0,042 \% \mathrm{KCl}, 0,024 \%$ $\mathrm{CaCl}_{2}$ und $0,03 \% \mathrm{NaHCO}_{3}$ und die Brom-Ringer aus $1,58 \% \mathrm{NaBr}$, $0,067 \% \mathrm{KBr}, 0,033 \% \mathrm{CaBr}_{2}$ und $0,03 \% \mathrm{NaHCO}_{3}$. Alle diese hier gebrauchten Salze waren immer Mercksche.

Aus Figg. 2, 3 und 4 ist ersichtlich, dass bei der Durchströmung der isolierten Gef:ässe, wenn die körperwarme Normal-Ringer nach konstant gewordenem Ausfluss auf Brom-Ringer umgeschaltet war, der Ausfluss sich rapid vermehrt und in mehreren Minuten sein Maximum erreicht, dann langsam auf den Wert vor der Umschaltung zurückkehrt oder langdauernd

Fig. 2 .

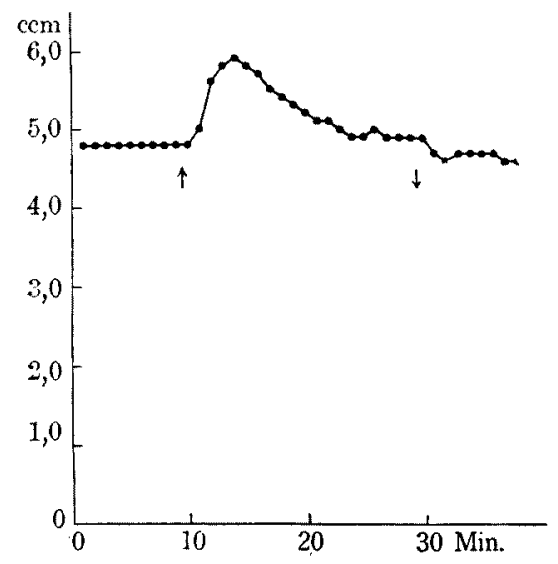

Durchströmung des isolierten Kaninchenschenkels. Bei $\uparrow$ Normal-Ringer auf Brom-Ringer und bei $\downarrow$ Brom-Ringer auf Normal-Ringer umgeschaltet.

Fig. 3.

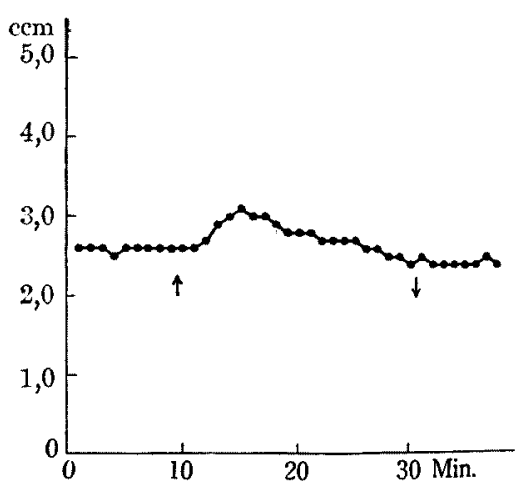

Durchströmung der isolierten Kaninchenohrgefïsse. Bei $\uparrow$ Brom-Ringer eingewirkt. 


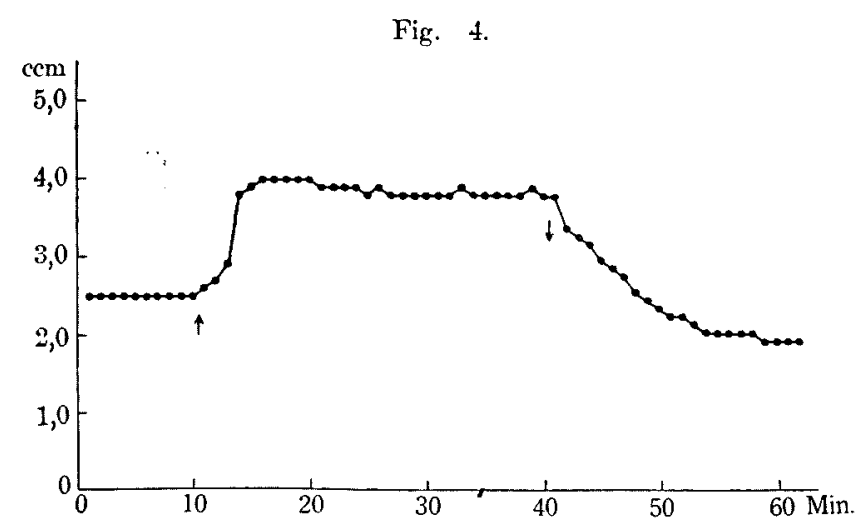

Durchströmung des isolierten Kaninchenschenkels.

Bei $\uparrow$ Prom-Ringer eingewirkt.

vermehrt bleibt. Auch bei der vergleichenden Untersuchung der durch Adrenalin bedingten Gefässverengerung an einem mit Normal- oder BromRinger durchspülten Gefässprüparat zeigen die beiden Lösungen keinen merklichen Unterschied, also auch keinen in der Empfindlichkeit des Sympathikus der Gefüsse.

Damit ist die crweiternde Wirkung des Broms auf die peripheren Gefässe klargestellt; von hier aus gesehen, rührt wohl in meinen Versuchen die im Vergleich zur NaCl-Lösung grössere Zunahme des Minutenvolums, die unmittelbar nach Injektion der Bromnatriumlösung beobachtet wurde, wenigstens zum Teil von der Erweiterung der peripheren Gef:isse durch $\mathrm{Br}$ her.

Unmittelbar nach der Injektion vermehrt sich das Minutenvolum, diese Vermehrung verschwindet im allgemeinen in 1 Stunde, und es nimmt nachweislich wieder im Versuch mit Chlornatrium etwas zu, aber im Versuch mit Bromnatrium auffallend ab. Das Minutenvolum verändert sich mit dem Gaswechsel, und der Sauerstoff verbrauch vermehrt sich, wie schon gesagt, ,7) beim Versuch mit $\mathrm{NaCl}$ etwas, vermindert sich aber im Versuch mit $\mathrm{NaBr}$ erheblich. Da im ersten Fall das Minutenvolum dem Sauerstofflkonsum entsprechend zunimmt, könnte sich diese Zunahme des Minutenvolums sekundär als Folge des vermehrten Sauerstoffverbrauchs geltend machen, während im letzten Fall die Abnahme des Minutenvolums nachweislich bei weitem bedeutender ist als die des Sauerstoffkonsum. Also lïsst sie sich schwer einfach dadurch allein erklären, dass sie anf dieser beruht. Nach Eulenburg und Guttmann, ${ }^{7)}$ Greene und Kruse, ${ }^{3)}$ darf 
die Sache so auffasscn : das Minutenvolum wird dadurch erheblich vermindert, dass $\mathrm{Br}$ durch direkte Wirkung auf das Herz seine Tätigkeit herabsetzt und dadurch seine dynamische Kraft schwächt. Die Pulsverlangsamung $1 \frac{1}{2}-2$ Stunden nach der Injektion der $\mathrm{NaBr}$-Lösung spricht für die obige Tatsache, d. h. die Herabsetzung der Herztätigkeit.

Aus all dem oben Erwähnten folgt, dass Brom, auch von Seiten des Minutenvolums aus betrachtet, die Herztätigkeit von Kaninchen herabsetzt.

Schlussfolgerung.

1. Durch intravenöse Injektion hypertonischer Chlor- oder Bromnatriumlösung bei Kaninchen vermehrt sich das Minutenvolum unmittelbar danach und zwar durch diese immer stärker als durch jene.

2. Anderthalb bis zwei Stunden nach intravenösem Einspritzen von Bromnatriumlösung nimmt das Minutenvolum erheblich ab.

3. Werden die Gefässe des isolierten Kaninchenschenkels und -ohrs mit Ringer durchströmt, deren sümtliches $\mathrm{Cl}$ durch $\mathrm{Br}$ ersetzt ist, so erweitern sie sich. 